\title{
Study on excimer laser irradiation for controlled dehydrogenation and crystallization of boron doped hydrogenated amorphous/nanocrystalline silicon multilayers
}

\author{
F. Gontad ${ }^{\text {a,* }}$, J.C. Conde ${ }^{\text {a }}$, S. Filonovich b, ${ }^{\text {, M.F. Cerqueira }}{ }^{\text {b }}$, P. Alpuim ${ }^{\text {b }}$, S. Chiussi ${ }^{\text {a }}$ \\ a Applied Physics Department, University of Vigo, E.I. Industrial, Campus de As Lagoas, Marcosende, E-36310, Vigo, Spain \\ ${ }^{\mathrm{b}}$ Department of Physics, University of Minho, Campus de Azurém, 4800-058 Guimarães, Portugal
}

\section{A R T I C L E I N F O}

\section{Article history:}

Received 20 June 2012

Received in revised form 2 April 2013

Accepted 5 April 2013

Available online 17 April 2013

\section{Keywords:}

Excimer laser annealing

Dehydrogenation

Hydrogenated amorphous silicon

Boron doped nanocrystalline silicon

\begin{abstract}
A B S T R A C T
We report on the excimer laser annealing (ELA) induced temperature gradients, allowing controlled crystallization and dehydrogenation of boron-doped a-Si:H/nc-Si:H multilayers. Depth of the dehydrogenation and crystallization process has been studied numerically and experimentally, showing that temperatures below the monohydride decomposition can be used and that significant changes of the doping profile can be avoided. Calculation of temperature profiles has been achieved through numerical modeling of the heat conduction differential equation. Increase in the amount of nano-crystals, but not in their size, has been demonstrated by Raman spectroscopy. Effective dehydrogenation and shape of the boron profile have been studied by time of flight secondary ion mass spectroscopy. The relatively low temperature threshold for dehydrogenation, below the monohydride decomposition temperature, has been attributed to both, the large hydrogen content of the original films and the partial crystallization during the ELA process. The results of this study show that UV-laser irradiation is an effective tool to improve crystallinity and dopant activation in $\mathrm{p}^{+}$-nc-Si:H films without damaging the substrate.
\end{abstract}

(c) 2013 Elsevier B.V. All rights reserved.

\section{Introduction}

Amorphous and nanocrystalline silicon thin film devices play an important role in the fabrication of large-area electronics, including flat panel displays, X-ray imaging devices and photovoltaic panels.

Currently, the most common thin film silicon deposition techniques are radio-frequency plasma enhanced chemical vapor deposition, and hot wire chemical vapor deposition (HWCVD) [1,4]. Although CVD techniques are well established and allow one to obtain Si films of high electronic quality, easily doped from gas phase with boron, phosphorus or arsenium using gaseous precursors of these elements, the films obtained have nevertheless some structural flaws. Typical nc-Si:H films consist of an amorphous incubation layer starting at the substrate interface, followed by a partially of fully crystallized region, depending on deposition conditions. This structure has some drawbacks for applications $[3,5,6]$. First, in devices like bottom gate thin-film transistors (TFTs), charge transport occurs in the channel that forms within the first few layers of the film, which are part of the incubation layer. Second, in many cases, like in the contacts of a TFT or in any of the solar cell layers, transport is perpendicular to the substrate, through the thickness of the film, thus involving the more

\footnotetext{
* Corresponding author at: Istituto Nazionale di Fisica Nucleare, Sezione di Lecce, Via per Arnesano, 73100 Lecce, Italy. Tel.: + 393806599695.

E-mail addresses: fran_gontad@yahoo.es, francisco.gontad@le.infn.it (F. Gontad).

1 Present address: CENIMAT/I3N-CEMOP, Universidade Nova de Lisboa, 2829-516 Caparica, Portugal.
}

defective incubation layer. Third, doped layers are normally used as contacts in devices, with thickness typically of a few tens of nanometers, thus being negatively affected by the incubation layer. Conventional thermal techniques cannot be used to crystallize the films or to activate the dopants because they would damage the low-temperature substrates used in large-area applications and they definitely prevent the use of thermo-sensible polymeric foils, that are used as substrates for flexible electronic applications. An alternative route that allows hydrogen content tailoring, improving crystallinity, and doping activation without heating up the entire sample, including the substrate is therefore of great interest.

$193 \mathrm{~nm}$ ELA (excimer laser annealing) can be considered as such an attractive choice because of its capabilities to ensure low thermal budgets, controlled penetration depth and localized processing. These facts are based on the properties of the pulsed ArF excimer laser radiation, with a wavelength of $193 \mathrm{~nm}$ and pulse duration of a few tens of nanoseconds that only involves the warm-up of very shallow near surface regions for a short time period [7]. Thus, the technique is also applicable in the case of using polymers and glasses as substrate materials, because the temperature reached on the substrate can be controlled by means of the laser fluence used $[8,9]$. Moreover, the temperature depth profile gradient achieved in this process can be previously calculated via numerical solutions of the heat differential equations, providing an estimation of the adequate laser fluence needed for annealing a sample without excessive heating of the substrate $[9,10]$. In addition, due to the ultrafast processes involved in ELA both, the diffusion of doping elements, 
the crystallization process and the effusion of hydrogen can be controlled, through the adjustment of the laser fluence dependent temperature profiles [7-9].

In the present work we report experimental studies on the $193 \mathrm{~nm}$ ELA induced modification of hydrogen content and crystallinity in multilayered structures with alternating amorphous and boron doped nano-crystalline silicon films that have been prepared by HWCVD at low substrate temperatures. The choice of such multilayered structures was based on the importance of both amorphous and nanocrystalline silicon in the electronic, photonic and photovoltaic industries and the large hydrogen content of those films deposited by HWCVD. In addition, those structures provide the possibility of analyzing not only hydrogen, but also dopant diffusion. Moreover, in order to select the most appropriate laser fluences, a previously developed [10] numerical simulation of the temperature profiles produced by the employed laser pulses was carried out. Special attention was focused on the study of the changes produced in boron doping profile and crystallinity of the multilayer.

\section{Experimental details}

Alternating $20 \mathrm{~nm}$ amorphous hydrogenated silicon (a-Si:H) and $80 \mathrm{~nm}$ boron doped hydrogenated nanocrystalline silicon (nc-Si:H) films were successively stacked on a Corning 7059 glass substrate by HWCVD with an equipment described elsewhere [11] at the same substrate temperature $\left(\mathrm{T}_{\mathrm{s}}=150{ }^{\circ} \mathrm{C}\right)$ and working pressure $\left(\mathrm{P}_{\mathrm{w}}=\right.$ of $\left.5.3 \mathrm{~Pa}\right)$, as can be seen in Fig. 1, where a schematic model and a SEM cross section of the multilayered structure are provided. Boron doped nc-Si:H films were deposited using hydrogen dilution, $H D$, of the reactive gas mixtures, consisting of silane $\left(\mathrm{SiH}_{4}\right)$ and diborane $\left(\mathrm{B}_{2} \mathrm{H}_{6}\right)\left[\mathrm{HD}=\mathrm{F}_{\mathrm{H}_{2}} /\left(F_{\mathrm{SiH}_{4}}+F_{\mathrm{H}_{2}}+F_{\mathrm{B}_{2} \mathrm{H} 6}\right) \times 100 \%\right.$, where the $F$ s are gas flow rates]. Different diborane/silane flow rate ratios $0.2 \leq R \leq 3.5, \quad\left(R=F_{\mathrm{B}_{2} \mathrm{H} 6} / F_{\mathrm{SiH}_{4}} \times 100 \%\right)$, were used in order to achieve different doping concentrations for each individual nc-Si:H film. In the growth of the buffer layers only silane without diborane nor hydrogen dilution were employed, to ensure the formation of an intrinsic hydrogenated amorphous silicon layer effectively separating the doped layers. The final structure consisted of 13 layers of a-Si:H and 12 layers of boron doped nc-Si:H stacked with a total stack thickness of approximately $1200 \mathrm{~nm}$. Hydrogen dilution was $H D=95 \%$ for the first eight $\mathrm{p}^{+}$-nc-Si:H layers and for layer \#11 (see Fig. 1). Two layers (\#9 and \#10) were deposited under lower $H D$ ( $80 \%$ and $90 \%$, respectively). The boron and $\mathrm{SiH}$ bond distribution along the whole as-deposited structure can be clearly seen in Fig. 2,

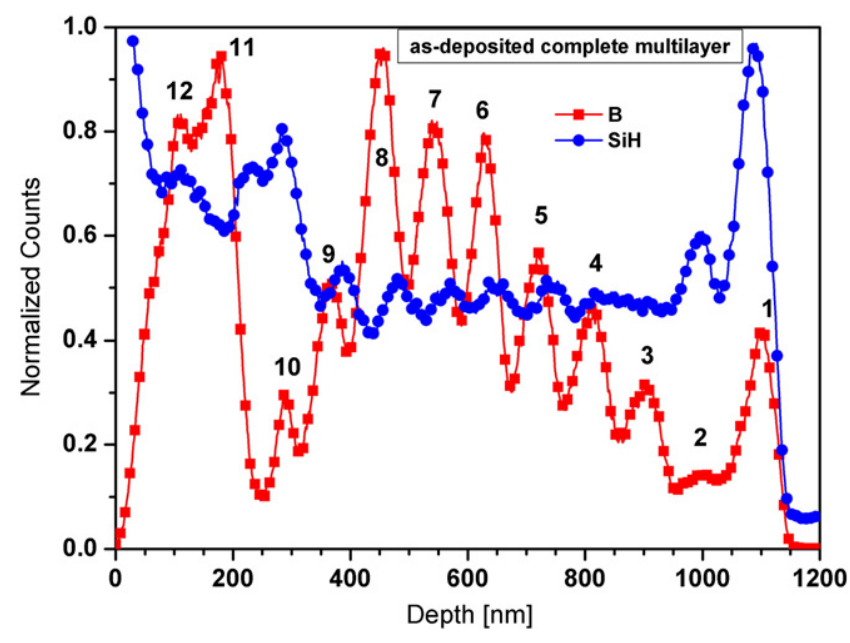

Fig. 2. TOF-SIMS depth profile of boron and $\mathrm{SiH}$ in the whole as-deposited multilayered structure. The numbers identifying the boron peaks correspond one-to-one to the numbers identifying the doped layers in the scheme of Fig. 1 (left side).

where its time of flight secondary ion mass spectroscopy depth profiles with the correspondent position of the 12 doped layers are provided.

The multilayer structure was irradiated in helium environment (99.9999\% purity) at room temperature and atmospheric pressure for performing ELA. During the experiments, the radiation of a $193 \mathrm{~nm}$ excimer laser (Lampda Physik LPX 220i), with a pulse length of around $25 \mathrm{~ns}$, impinged normally to the sample surface. The beam intensity was spatially homogenized using a fly-eye homogenizing system (Exitech Limited Beam homogenizer EX-HS-700D) and "in situ" monitored (Ophir PE50-DIF) as described elsewhere [12]. Various areas of $8.8 \mathrm{~mm}^{2}$ were irradiated with different laser fluencies (70-300 mJ/ $\mathrm{cm}^{2}$ ) and number of pulses (1 and 10).

For studying the dehydrogenation process through laser annealing and its possible influence in the boron doping profile, time of flight secondary ion mass spectrometer (TOF-SIMS) and Raman analysis have been performed. Depth profiling analysis was carried out with a TOF-SIMS IV (Ion-TOF) and achieved sputtering the sample with an $\mathrm{O}_{2}$ gun with a $10 \mathrm{kV}$ voltage while a pulsed $\mathrm{Ga}^{+}$beam was used for extracting the secondary ions. Raman measurements were performed with a Jobin Yvon T6400 using an excitation wavelength of $488 \mathrm{~nm}$ with a fluence well below the crystallization threshold of
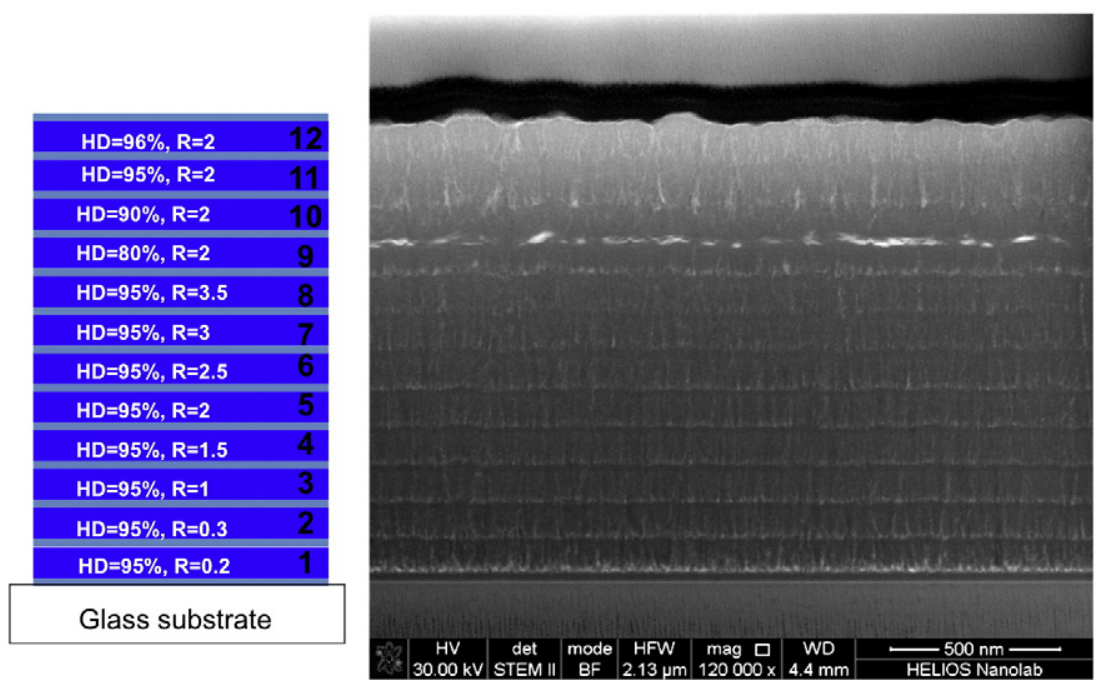

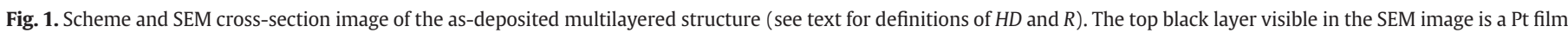
used for protection during the sample preparation by focused ion beam. 
the amorphous silicon in order to avoid Raman laser induced changes of crystallinity and grain size.

\section{Numerical simulation}

The calculation of the temperature induced by irradiating the multilayered structure was performed for two models with different thicknesses of a-Si:H and nc-Si:H films. The aim of using two different models was to estimate the influence of possible fluctuations in the real thickness of the samples grown by HWCVD. In the first case, a thickness of $25 \mathrm{~nm}$ for a-Si:H films and $100 \mathrm{~nm}$ for nc-Si films was considered, until a total thickness of $900 \mathrm{~nm}$. In the other one, the thickness was 30 and $60 \mathrm{~nm}$ for a-Si:H and nc-Si:H, respectively, being again $900 \mathrm{~nm}$ the total thickness. In both cases, Corning glass was considered as substrate and laser fluence to be in the range from 50 to $300 \mathrm{~mJ} / \mathrm{cm}^{2}$.

The temperature distribution achieved by the ArF excimer laser in both models has been calculated by solving the heat conduction differential equation [13] with the finite element method and using the commercial software ANSYS@ (10.0) [14], as described in previous works $[15,16]$. The collection of equations, boundary and initial conditions in the nodes of a mesh previously defined used for this calculation is extensively described in [10]. While the dependence of the material properties $k(T), \rho(T)$ and $C(T)$ on the temperature [17-19] has been taken into account, the losses of heat by convection and radiation have not been considered.

\section{Results and discussion}

The temperature distribution calculated for both models is shown in Fig. 3. The most representative laser fluences are plotted for every model. In addition, the decomposition temperature for mono-, diand trihydrides and a-Si melting point are marked on the figure for a better understanding of the achieved results. Comparing Fig. 3a (sample with $100 \mathrm{~nm}$ nc-Si layers) with Fig. 3b (sample with $60 \mathrm{~nm}$ nc-Si layers) one can easily observe that the model predicts for identical fluences with different depths of temperature thresholds. Thresholds for thinner nc-Si layers are reached in deeper regions than for thicker nc-Si layers. This is consistent with the fact that the multilayer with the $60 \mathrm{~nm}$ nc-Si has a larger fraction of a-Si:H, thus releases more latent heat during crystallization [9].

For evaluating the depth to which dehydrogenation can be achieved, TOF-SIMS depth profile analysis of $\mathrm{SiH}$ fragments has been acquired. Fig. 4 shows the normalized spectra, indicating the presence of hydrogen bonded to silicon in the upper layers of the structure, where changes after laser irradiation are produced. Specifically, the profile of the structure, as-deposited and after being irradiated with one laser pulse and laser fluences of 70,140 and $200 \mathrm{~mJ} / \mathrm{cm}^{2}$, respectively, down to a depth of $550 \mathrm{~nm}$ is presented. As can be seen, the $\mathrm{SiH}$ signal is decreasing significantly down to a depth of $170 \mathrm{~nm}$ when the sample is irradiated with a fluence of $70 \mathrm{~mJ} / \mathrm{cm}^{2}$, while no significant differences compared to the as-deposited multilayer can be detected in deeper zones. Higher fluences, such as 140 and $200 \mathrm{~mJ} / \mathrm{cm}^{2}$, provoke an even stronger decrease in the $\mathrm{SiH}$ signal. An interesting effect can be observed for the highest fluence at depths between 40 and $140 \mathrm{~nm}$ where the $\mathrm{Si}-\mathrm{H}$ signal is higher than expected, when compared with the corresponding signal from deeper zones. This can be attributed to the recombination of silicon with hydrogen diffusing from deeper zones to the surface. Part of the hydrogen atoms that have been removed from deeper regions seem to find the silicon recombination centers and link to them, thus avoiding the complete effusion from the upper layers. However, it can be assumed that the majority of the hydrogen is effusing and recombining to $\mathrm{H}_{2}$ molecules during the diffusion process [20].

In the calculated simulation of the ELA, the dehydrogenation of the multilayer at fluences near $100 \mathrm{~mJ} / \mathrm{cm}^{2}$ is expected to occur down to
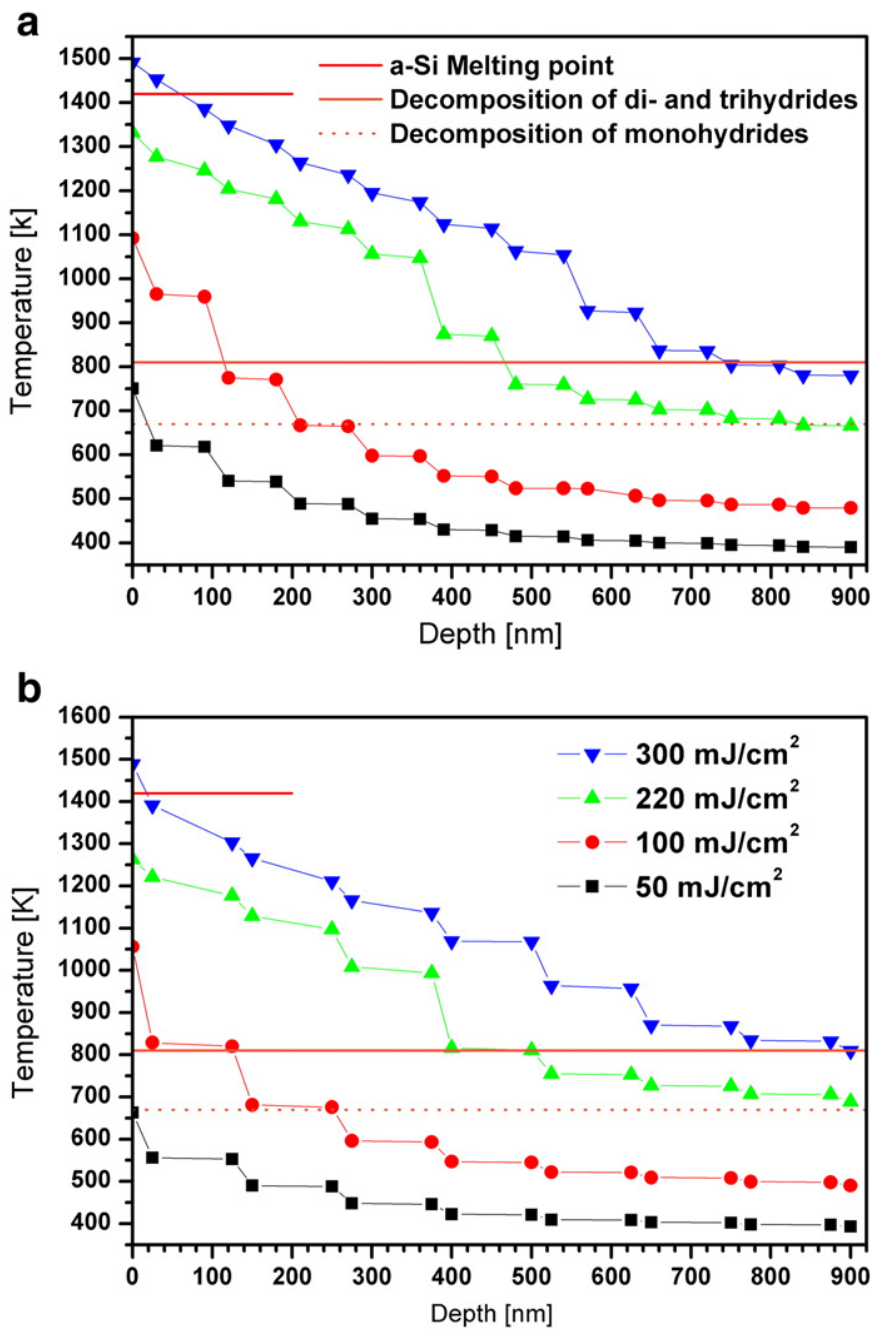

Fig. 3. Calculated temperature distribution for the multilayered structure with a film thickness of: a) $25 \mathrm{~nm}$ and $100 \mathrm{~nm}$ for a-Si:H and nc-Si:H, respectively and b) $30 \mathrm{~nm}$ and $60 \mathrm{~nm}$ for a-Si:H and nc-Si:H.

an approximate depth of $130 \mathrm{~nm}$ for both models, considering that the effective dehydrogenation occurs when the monohydride phases are starting to decompose $(810 \mathrm{~K})$ [21]. Taking this threshold into account one should not expect to produce dehydrogenation down to

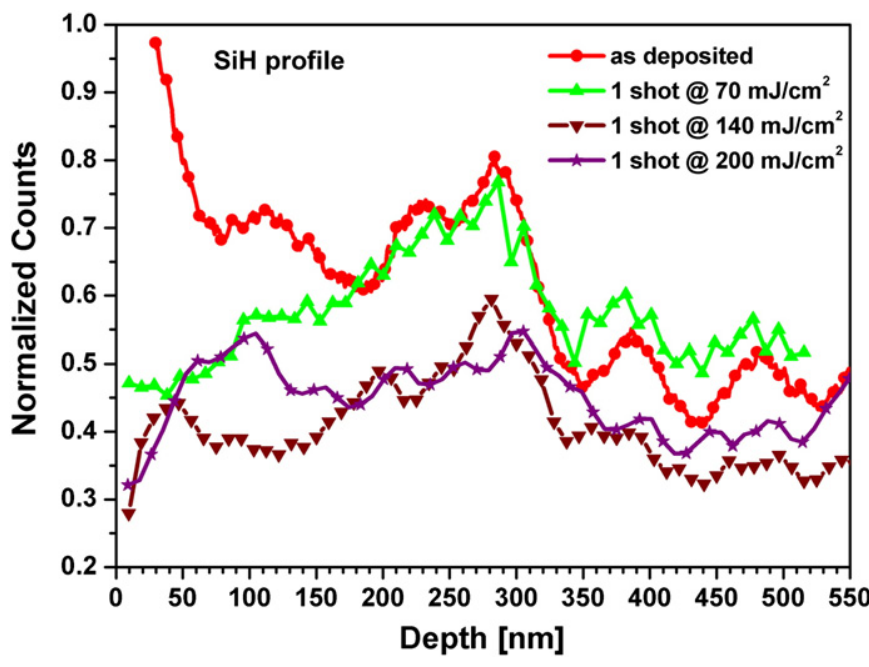

Fig. 4. TOF-SIMS depth profile of $\mathrm{SiH}$ for different laser fluences down to a depth of $550 \mathrm{~nm}$. 
the depth that has been actually achieved in the experiments. For a laser fluence of $70 \mathrm{~mJ} / \mathrm{cm}^{2}$, a dehydrogenation of the film down to a depth of $100 \mathrm{~nm}$ has been predicted, but the amount of hydrogen present in the film decreased from the top surface down to $170 \mathrm{~nm}$. The rather large difference between simulation and experimental results can be attributed to several reasons. The lower deposition temperature compared to typical a-Si:H producing a higher amount of $\mathrm{H}$ bonds and the boron doping itself, decrease the dehydrogenation temperature more than $100 \mathrm{~K}$ [20]. In addition, the studied HWCVD nc-Si:H films are not completely crystalline, showing a crystalline fraction that strongly depends on the ratio of diborane/silane [11]. Thus, the annealing and subsequent crystallization of the amorphous matrix in the nc-Si:H films can produce an additional amount of latent heat [9] that has not been considered in the simulation. The release of this additional latent heat provides a supplementary increase of temperature in the structure and, hence, the dehydrogenation of the structure down to deeper zones. Taking these considerations into account, the temperature distribution obtained by numerical simulation agrees with the changes of the $\mathrm{SiH}$ signal found after laser annealing.

The experimental results indicate that, as expected, crystallization of the laser treated volume occurs. Raman analysis using $488 \mathrm{~nm}$ excitation wavelength, performed keeping the excitation laser beam intensity well below the crystallization threshold, indicates that the amorphous $\mathrm{Si}-\mathrm{Si}$ band around $480 \mathrm{~cm}^{-1}$ decreases, while a relatively broad peak around $514 \mathrm{~cm}^{-1}$ clearly increases in intensity with the ELA treatment (Fig. 5). This peak at $514 \mathrm{~cm}^{-1}$, with a full width at half maximum between 18 and $20 \mathrm{~cm}^{-1}$, characteristic for the as-deposited nc-Si:H films, can be attributed to nanocrystalline $\mathrm{Si}$ with crystallite sizes of around $5 \mathrm{~nm}$ [22] and increases its intensity even when the sample is irradiated with only one shot at $70 \mathrm{~mJ} / \mathrm{cm}^{2}$. Moreover, the fact that this peak does not significantly shift with higher laser energy densities indicates that the crystallite sizes do not significantly change. The absence of a Si-Si peak at $521 \mathrm{~cm}^{-1}$, like the one obtained by measuring a $\mathrm{Si}(100)$ wafer as reference, or peaks at wave numbers between 514 and $520 \mathrm{~cm}^{-1}$, confirms the absence of larger crystals. Furthermore, a reduction of peak width with the amount of laser pulses is also evident, as shown in a comparison of Raman spectra between one and ten laser shots at 70 and $300 \mathrm{~mJ} / \mathrm{cm}^{2}$ (Fig. 5). The shoulder of the nc-Si, caused by a broad band centered at $480 \mathrm{~cm}^{-1}$, assigned to the amorphous fraction of the multilayered sample, decreases with the number of laser pulses. In addition, its integrated area does not seem to be significant than that for one laser pulse. This fact evidences an increase in the crystalline fraction with the laser fluence and number of pulses as can be also noted in Table 1, where the crystalline fraction of the Raman
Table 1

Crystalline fraction of the as-deposited and irradiated samples.

\begin{tabular}{lll}
\hline & Crystalline fraction, $\mathrm{X}_{\mathrm{c}}(\%)$ & \\
\hline As-deposited & 56 & 10 laser shots \\
Irradiated samples & 1 laser shot & 78 \\
$70 \mathrm{~mJ} / \mathrm{cm}^{2}$ & 72 & 79 \\
$140 \mathrm{~mJ} / \mathrm{cm}^{2}$ & 74 & 98 \\
$300 \mathrm{~mJ} / \mathrm{cm}^{2}$ & 92 & \\
\hline
\end{tabular}

probed volume has been calculated according to Ledinsky et al. [23]. It should be noted that for all samples the Raman signal is collected from an interaction volume whose depth largely exceeds the thickness of one single layer. If one assumes that at the $455 \mathrm{~nm}$ Raman excitation wavelength the amorphous silicon optical absorption coefficient $\alpha \sim 10^{4}-10^{5} \mathrm{~cm}^{-1}$ then the light penetration depth in the sample $\sim 1 / \alpha$ is in the range $100-1000 \mathrm{~nm}$. The dramatic increase in crystalline fraction upon laser irradiation can most likely be attributed not only to crystallization of the amorphous layers but also of the amorphous matrix in the nc-Si:H layers. This strong increase of crystalline fraction (going from $X_{c}=56 \%$ in as deposited sample to $X_{c}=72 \%$ after 1 laser shot at $70 \mathrm{~mJ} / \mathrm{cm}^{2}$ until $X_{c}=98 \%$ after 10 shots at $300 \mathrm{~mJ} / \mathrm{cm}^{2}$ ) can also explain the differences found between experimental results and predicted numerical simulations. As dehydrogenation occurs down to deeper zones than predicted by simulations, an additional energy supply must exist, contributing to this stronger dehydrogenation.

Another interesting issue is, if dehydrogenation and crystallization in such kind of structures can be performed without causing significant boron diffusion and, to which depth this can be done. TOF-SIMS depth profile signal of boron for as-deposited multilayer and its evolution after irradiating the sample with different laser fluences have therefore been plotted in Fig. 6 , showing the normalized boron counts. For the lowest fluences ( 70 and $140 \mathrm{~mJ} / \mathrm{cm}^{2}$ ) a slight decrease of the boron signal, observed from the top surface (down to a depth of $180 \mathrm{~nm}$ ), might be attributed to the matrix effect produced by the different crystallinity of the measured spots. Apart from these issues, both signals seem to be quite similar, in particular the position and shape of the interfaces between doped and undoped layers.

On the contrary, significant changes were observed for a laser fluence of $200 \mathrm{~mJ} / \mathrm{cm}^{2}$, where the interface between doped and undoped layers seems to be less sharp and slightly shifted. Combining the features that can be seen in Figs. 2 and 4, we can conclude that with increasing laser fluence up to $140 \mathrm{~mJ} / \mathrm{cm}^{2}$, hydrogen is effectively removed from the film without varying significantly the doping profile.

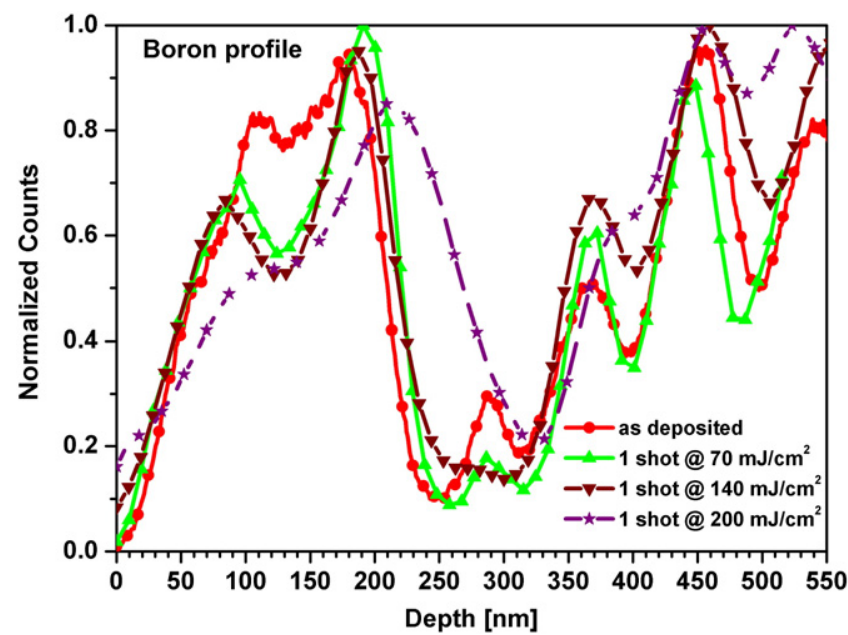

Fig. 6. TOF-SIMS depth profile of boron doping for different laser fluences down to a depth of $550 \mathrm{~nm}$. 
For higher fluences, an effective dehydrogenation is still achieved, but changes in boron doping profile are also evident. In fact, for the highest laser fluence, $300 \mathrm{~mJ} / \mathrm{cm}^{2}$, the melting and recrystallization of the upper layers provoke degradation on the boron doping profile. Despite the short time of the laser annealing, boron diffuses along the upper molten layers deteriorating considerably the initial sharp profile. The latent heat released by crystallization of a-Si:H and the amorphous matrix of nc-Si:H, corroborated by the huge increase of crystalline fraction, produces a deeper annealed volume with lower hydrogen content than predicted by numerical simulations. Despite the differences between theoretical and experimental annealing depth, the simulation clearly indicates that dehydrogenation in these kind of hydrogen rich a-Si:H/ nc-Si structures should mainly be driven by decomposition of di- and trihydrides. Boron diffusion can, on the other hand, be avoided when working under a fluence threshold of $200 \mathrm{~mJ} / \mathrm{cm}^{2}$ that limits the maximum temperature in the volume.

\section{Conclusions}

Energy threshold for an efficient dehydrogenation of the a-Si:H/boron doped nc-Si:H multilayer avoiding drastic changes in doping profile through excimer laser annealing has been determined. Laser fluences up to $300 \mathrm{~mJ} / \mathrm{cm}^{2}$, promote a partial crystallization of the amorphous structures with crystal grain sizes around $5 \mathrm{~nm}$ and without significant change in grain size of the nc-Si films. Partial crystallization of the amorphous matrix in the nc-Si layers takes also place, releasing additional latent heat and leading to a deeper annealed volume than predicted through typical numerical simulation. Fluence of $140 \mathrm{~mJ} / \mathrm{cm}^{2}$ is high enough to dehydrogenate the multilayer without significant changes in doping profile and reaching crystalline fraction of $74 \%$.

Using this study as a guide it should be possible to transfer the present results to films deposited on plastic substrates at very low substrate temperature $\left(\leq 150{ }^{\circ} \mathrm{C}\right)$ and improve by UV-laser irradiation their crystallinity and, for p-type films, the boron activation, without damaging the substrate.

\section{Acknowledgments}

This work has been partially supported by the following National and Regional research contracts: MAT-2000-1050, MAT-2003-04908,
MAT-2011-24077, PGIDIT03-04908, PGIDT-01PX130301PN, XUGAInfra 93, XUGA-Infra 94-58, SB93-A0742819D and INFRA 99-PR 405a-46, and the Spanish-Portuguese bilateral agreement HP20060122. Fundação para a Ciência e Tecnologia (FCT) is acknowledged for the postdoctoral grant of S. Filonovich.

\section{References}

[1] C.A. Wolden, J. Kurtin, J.B. Baxter, I. Repins, S.E. Shaheen, J.T. Torvik, A.A. Rockett, V.M. Fthenakis, E.S. Aydil, J. Vac. Sci. Technol. A 29 (3) (2011) 030801.

[2] EPIA, 2010.

[3] B. Rech, O. Kluth, T. Repmann, T. Roschek, J. Springer, J. Müller, F. Finger, H. Stiebig, H. Wagner, Sol. Energy Mater. Sol. Cells 74 (2002) 439.

[4] K.L. Choy, Prog. Mater. Sci. 48 (2001) 57.

[5] G.B. Tong, S.A. Rahman, Effects of Substrate Temperature on the Properties of Hydrogenated Nanocrystalline Silicon Thin Films Grown by Layer-by-Layer Technique, Kuala Lumpur, Malaysia, October 29-December 1, IEEE Int. Conf. on Semicond. Electron. Proc., ICSE06, 2006, p. 472.

[6] W.M.M. Kessels, A.H.M. Smets, D.C. Marra, E.S. Aydil, D.C. Schram, M.C.M. van de Sanden, Thin Solid Films 383 (2001) 154

[7] E.V. Monakhov, B.G. Svensson, M.K. Linnarsson, A. La Magna, V. Privitera, M. Camalleri, G. Fortunato, L. Mariucci, Mater. Sci. Eng. B 114-115 (2004) 353.

[8] V. Privitera, S. Scalese, A. La magna, A. Pecora, M. Cuscunà, L. Maiolo, A. Minotti, D. Simeone, L. Mariucci, G. Fortunato, L. Caristia, F. Mangano, S. Di Marco, M. Camalleri, S. Ravesi, S. Coffa, M.G. Grimaldi, R. De Bastiani, P. Badal, S. Bagiante, J. Electrochem. Soc. 155 (2008) 764.

[9] F. Falk, G. Andrä, J. Cryst. Growth 287 (2006) 397.

[10] L. Fornarini, J.C. Conde, S. Chiussi, F. Gontad, B. León, S. Martelli, Appl. Surf. Sci. 254 (2007) 898.

[11] P. Alpuim, M. Andrade, V. Sencadas, M. Ribeiro, S.A. Filonovich, S. Lanceros-Mendez, Thin Solid Films 515 (2007) 7658.

[12] E. López, S. Chiussi, J. Serra, P. González, B. León, Thin Solid Films 508 (2006) 48.

[13] L. Calcagnile, M.G. Grimaldi, P. Baeri, J. Appl. Phys. 76 (3) (1994) 1833.

[14] ANSYS $®$, Analysis Guides and others, First Edition, SAS IP, Inc. @ 2005.

[15] J.C. Conde, P. González, F. Lusquiños, S. Chiussi, J. Serra, B. León, Appl. Surf. Sci. 248 (2005) 455.

[16] J.C. Conde, P. González, F. Lusquiños, S. Chiussi, J. Serra, B. León, Appl. Surf. Sci. 248 (2005) 461.

[17] S. De Unamuno, E. Fogarassy, Appl. Surf. Sci. 36 (1989) 1.

[18] In: E.D. Palik (Ed.), Handbook of Optical Constants of Solids, Academic Press, Inc., USA, 1985.

[19] L. Ward, The Constants of the Materials and Films, second ed. IOP Publishing Ltd Institute of Physics, London, 1994.

[20] W. Beyer, Sol. Energy Mater. Sol. Cells 78 (2003) 235.

[21] J.J. Boland, Phys. Rev. B 44 (1991) 1383.

[22] Ch. Ossadnik, S. Veprek, I. Gregora, Thin Solid Films 337 (1999) 148.

[23] M. Ledinsky, L. Fekete, J. Stuchlik, T. Mates, A. Fejfar, J. Kocka, J. Non-Cryst. Solids 352 (2006) 1209. 